\title{
Targeting COVID-19 Viral Enzymes in an Evolving Landscape of Publishing and Peer Review
}

\author{
James Fraser \\ UCSF, San Francisco, United States of America; \\ jaimefraser@gmail.com
}

The severe acute respiratory syndrome coronavirus 2 (SARS-CoV-2) macrodomain within the nonstructural protein 3 counteracts host-mediated antiviral adenosine diphosphate-ribosylation signaling. This enzyme is a promising antiviral target because catalytic mutations render viruses nonpathogenic. We conducted a massive crystallographic screening and computational docking effort, identifying new chemical matter primarily targeting the active site of the macrodomain. X-ray data collection to ultra-high resolution and at physiological temperature enabled assessment of the conformational heterogeneity around the active site. Neutron diffraction data is guiding hydrogen placement to improve docking calculations. Several hits have promising activity in solution and provide starting points for development of potent SARS-CoV-2 macrodomain inhibitors. The role of entropy in modulating binding affinity will also be discussed.

Keywords: SARS, entropy, fragments, X-ray crystallography 